\title{
ABOUT THE CHOICE OF THE VARIABLE TO UNASSIGN IN A DECISION REPAIR ALGORITHM
}

\author{
CÉdric Pralet ${ }^{1}$ And GÉrard Verfaillie ${ }^{1}$
}

\begin{abstract}
The decision repair algorithm (Jussien and Lhomme, Artificial Intelligence 139 (2002) 21-45), which has been designed to solve constraint satisfaction problems (CSP), can be seen, either (i) as an extension of the classical depth first tree search algorithm with the introduction of a free choice of the variable to which to backtrack in case of inconsistency, or (ii) as a local search algorithm in the space of the partial consistent variable assignments. or (iii) as a hybridisation between local search and constraint propagation. Experiments reported in Pralet and Verfailllie (2004) show that some heuristics for the choice of the variable to which to backtrack behave well on consistent instances and that other heuristics behave well on inconsistent ones. They show also that, despite its a priori incompleteness, decision repair, equipped with some specific heuristics, can solve within a limited time almost all the consistent and inconsistent randomly generated instances over the whole constrainedness spectrum. In this paper, we discuss the heuristics that could be used by decision repair to solve consistent instances, on the one hand, and inconsistent ones, on the other hand. Moreover, we establish that some specific heuristics make decision repair complete.
\end{abstract}

Keywords. Constraint satisfaction problem, depth first tree search, local search, constraint propagation, backtrack, heuristics, completeness.

\section{INTRODUCTION}

In [8], Jussien and Lhomme proposed an algorithm called decision repair (or path repair in previous papers) dedicated to the solving of constraint satisfaction problems (CSP) $[4,9]$. This algorithm can be assessed along various points of view:

- it can be seen as an extension of the classical depth first tree search algorithm, allowing the variable to which to backtrack to be freely chosen

Received March 5, 2004. Accepted February 8, 2005.

${ }^{1}$ LAAS-CNRS, Toulouse, France; cpralet@laas.fr; gverfail@laas.fr

(C) EDP Sciences 2005 
among the currently assigned variables in case of inconsistency of the current partial assignment;

- it can also be seen as a local search algorithm in the space of the partial locally consistent variable assignments, with two kinds of move: variable assignment in case of local consistency and variable unassignment in case of inconsistency;

- it can finally be seen as a hybrid algorithm which closely combines local search and constraint propagation, the same way as usual constraint solving algorithms closely combine tree search and constraint propagation.

In this algorithm, value removal explanations, which are produced and recorded by constraint propagation, are sets of variables the assignment of which forbids a value $^{1}$. They play three roles:

- they allow an inconsistency explanation to be built when the domain of a variable becomes empty; this explanation is the union of the removal explanations of all the values of the variable the domain of which is empty; it means that the current assignment of all the variables involved in the explanation is inconsistent with the whole problem, and it can be exploited by choosing among these involved variables the one to unassign;

- they may allow an empty inconsistency explanation, that is a proof of inconsistency, to be built in case of inconsistency of the whole problem;

- they allow decremental constraint propagation to be performed efficiently in case of variable unassignment, without computing everything again from scratch.

With regard to termination, correctness, and completeness, its properties are the following:

- decision repair may not terminate, unless an arbitrary stopping criterion is implemented;

- it is correct: when solving a problem $P$, if it returns yes and a complete assignment $A, P$ is consistent and $A$ is solution of $P$; if it returns $n o, P$ is inconsistent;

- it is incomplete: when solving a problem $P$, consistent or not, it may never terminate; if the stopping criterion is activated, it returns ?, which means "I don't know".

Experiments reported in [10] lead to the following observations:

- decision repair may be far more efficient than usual local search algorithms, which search for a solution in the space of the complete variable assignments, and use no constraint propagation, only constraint checking;

- it may be more efficient than complete comparable algorithms such as chronological backtracking (usual depth first tree search), conflict directed backjumping [12], or dynamic backtracking [5];

\footnotetext{
1 In the decision repair algorithm, value removal explanations are more generally sets of decisions (domain restriction, constraint adding, etc.) which forbid a value. In this paper, we restrict ourselves to decisions that are value assignments.
} 
- it can solve many inconsistent problems at the frontier or beyond the frontier between consistency and inconsistency; within a limited time, it can even solve more inconsistent problems than complete comparable algorithms can;

- the heuristics used for the choice of the variable to unassign in case of inconsistency that are efficient to produce solutions and prove problem consistency are not the same as the ones that are efficient to prove problem inconsistency.

These observations led us to consider two questions:

(1) At least concerning the choice of the variable to unassign in case of inconsistency, are there heuristics dedicated to consistent problems and other ones dedicated to inconsistent ones, and which are they?

(2) Knowing that dynamic backtracking is an instance of decision repair where the variable to unassign is systematically the most recently assigned one in the current inconsistency explanation, but is complete, do there exist other or weaker conditions on the way of choosing the variable to unassign that guarantee completeness?

This paper presents preliminary answers to both questions. It is structured as follows: in Section 2, we present the decision repair pseudo code and, as examples, two traces of execution on a consistent problem and an inconsistent one; in Section 3, we show that there is no reason to use different heuristics for the choice of the variable to assign and the value to assign to it according to whether the problem is consistent or not; in Section 4, we show that the situation is different for the choice of the variable to unassign: various reasons lead to different heuristics according to the supposed nature of the problem; in Section 5, we present results of experiments carried out on randomly generated, but not completely homogeneous, consistent or inconsistent problems, with unassignment heuristics dedicated to consistent problems and dedicated to inconsistent ones; in Section 6 , we present two sufficient conditions for the completeness of the algorithm that generalise the proof of completeness of dynamic backtracking; finally, in Section 7, we list unanswered questions that deserve further attention.

\section{The DECISION REPAIR ALGORITHM}

\subsection{Algorithm Pseudo CODE}

The algorithm we consider is derived from the one presented in [8], but can be seen as a more generic version where more parameters remain to be set. Its pseudo code is shown in Figure 1.

The starting assignment $A$ may be empty, partial, or complete. Function Initial_Filter uses constraint propagation to enforce any local consistency property on the problem $P$ restricted by $A$. It returns True if $P$ is locally consistent and False if not. Function Extend_Assignment chooses a variable $v$ which is not assigned in the current assignment $A$ and extends $A$ by choosing a value $a$ for $v$. 


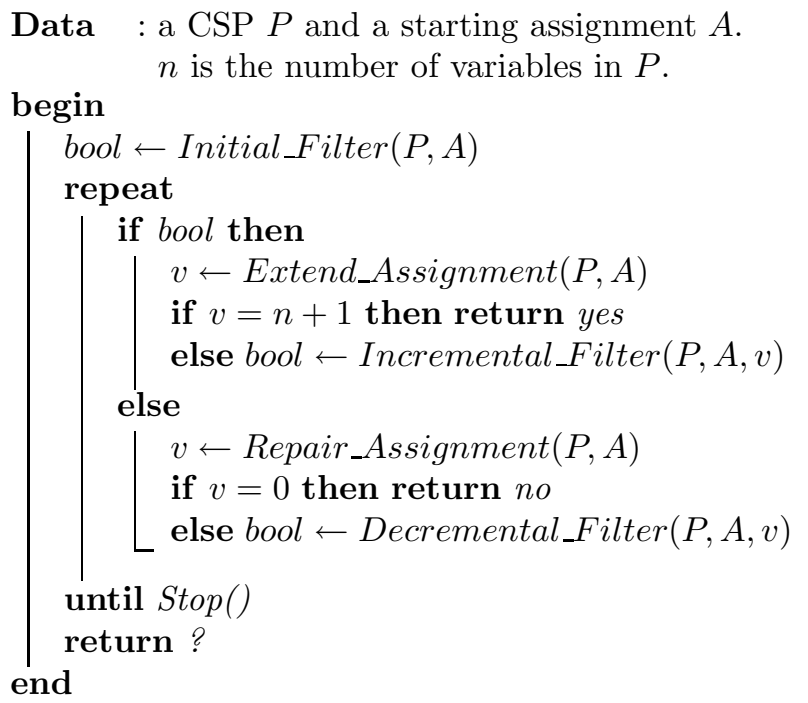

Figure 1. A generic decision repair algorithm.

It returns $n+1$ if there is no such variable. It is the case when $A$ is complete. Conversely, function Repair_Assignment chooses a variable $v$ which is assigned in the current assignment $A$ and repairs $A$ by unassigning $v$. It returns 0 if there is no such variable. It is the case when $A$ is empty or when inconsistency explanations are produced and recorded and the current inconsistency explanation is empty. Functions Incremental_Filter and Decremental_Filter use respectively incremental and decremental algorithms to enforce local consistency, without computing everything again from scratch. As does Initial_Filter, they return True if the current subproblem is locally consistent and False if not. Function Stop implements any stopping criterion.

\subsection{Algorithm outputs}

The algorithm ends with a proof of consistency of $P$ and an associated solution (answer yes), with a proof of inconsistency of $P$ (answer no), or with nothing in case of activation of the stopping criterion (answer ?). See Figure 2 for a comparison with the outputs of classical tree or local searches. Note the complete symmetry of the algorithm with regard to extension and repair and to consistency and inconsistency.

\subsection{Algorithm MAin PARAmeters}

The main parameters that remain to be set are the following ones:

- the local consistency property which is enforced at each step of the algorithm and the way of enforcing it incrementally or decrementally: forward checking, arc consistency, etc. 


\begin{tabular}{r|c|c} 
& Consistent instances & Inconsistent instances \\
\hline Tree search & $Y e s$ & No \\
\hline Local search & $Y e s$ or ? & ? \\
\hline Decision repair & $Y e s$ or ? & No or ?
\end{tabular}

Figure 2. Possible outputs on consistent and inconsistent problem instances.
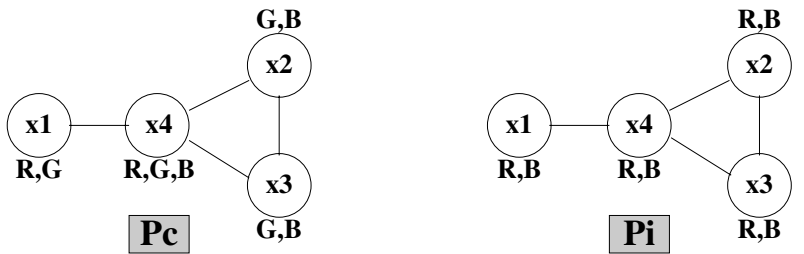

Figure 3. A consistent graph colouring problem $P_{c}$ and an inconsistent one $P_{i}$.

- the way value removal explanations are handled i.e., produced, recorded, combined, removed, etc.

- the variables and values that are affected by local consistency enforcing: all the variables or only the not assigned ones, all the values or only the currently not removed ones;

- the heuristics that are used in case of local consistency of the current partial assignment to choose the variable to assign and the value to assign to it;

- the heuristics that are used in case of inconsistency of the current partial assignment to choose the variable to unassign;

- the presence or absence of priority for assignment (resp. unassignment) to the variable that has been unassigned (resp. assigned) just before, when this assignment (resp. unassignment) immediately follows an unassignment (resp. assignment);

- finally, the stopping criterion which is used when no result (yes or no) has been produced.

\subsection{TWO TRACE EXAMPLES}

To put things in a more concrete form, we show in Figures 4 and 5 possible traces of such an algorithm on a consistent graph colouring problem $P_{c}$ and on an inconsistent one $P_{i}$ (see Fig. 3).

For these examples, we consider a specific decision repair algorithm which behaves as follows. After each assignment of a variable $v$, forward checking is performed from $v$ to the current domains of the not assigned variables. For each removed value, the singleton $\{v\}$ is recorded as a removal explanation. When the 


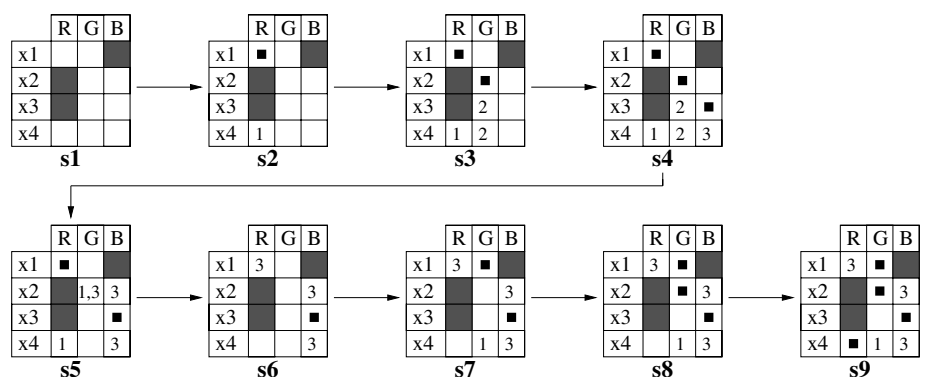

Figure 4. A possible execution of decision repair on $P_{c}$.

domain of a variable $v^{\prime}$ is wiped out, a variable $v^{\prime \prime}$ is chosen to be unassigned in the current inconsistency explanation, that is in the union of the value removal explanations in the domain of $v^{\prime}$. After unassignment of $v^{\prime \prime}$, a value removal explanation is created for its previous value (the previous inconsistency explanation minus $v^{\prime \prime}$ ). Irrelevant value removal explanations, those that involve $v^{\prime \prime}$, are then removed. The associated values are restored. But forward checking must be performed again from the assigned variables to the current domains of the not assigned ones. For assignment, the variable of lowest index among the variables of smallest current domain is chosen. Values are tried in the order $\{R, G, B\}$ for $P_{c}$ and $\{R, B\}$ for $P_{i}$. For unassignment, a variable is randomly chosen in the current inconsistency explanation.

Because decision repair in not a tree search but a local search in the space of partial assignments, its trace is only a sequence of states, each state being composed of a partial assignment and of a set of value removal explanations. In each state, initially forbidden values are pointed out in dark grey, current assignments by a small black square, and currently removed values by the indices of the variables that are involved in their removal explanation. Values the removal explanation of which is empty, those that are inconsistent whatever the assignment of the other variables is, are pointed out in light grey.

For example, on $P_{c}$ (see Fig. 4), in state $s_{4}$, the domain of variable $x_{4}$ is wiped out and all the other variables are involved in the inconsistency explanation. We assume that variable $x_{2}$ is chosen to be unassigned. This is what is done in state $s_{5}$. Value $G$ is removed from the domain of $x_{2}$ with $\left\{x_{1}, x_{3}\right\}$ as an explanation. The value removal explanations in which $x_{2}$ was involved are forgotten and associated values restored: value $G$ for $x_{3}$ and $x_{4}$. But forward checking the current domain of $x_{2}$ removes value $B$ with $\left\{x_{3}\right\}$ as an explanation.

On $P_{i}$, in state $s_{6}$ (see Fig. 5), the domain of variable $x_{4}$ is wiped out with $\left\{x_{2}\right\}$ as an explanation. Variable $x_{2}$ is unassigned and its previous value $R$ is removed with an empty explanation. It is thus sure that value $R$ for $x_{2}$ does not take part to any solution and can be removed from its domain. Similarly, when, in state $s_{10}$, the domain of variable $x_{3}$ is also wiped out with $\left\{x_{2}\right\}$ as an explanation, variable $x_{2}$ is unassigned and its previous value $B$ is removed with an empty explanation. 


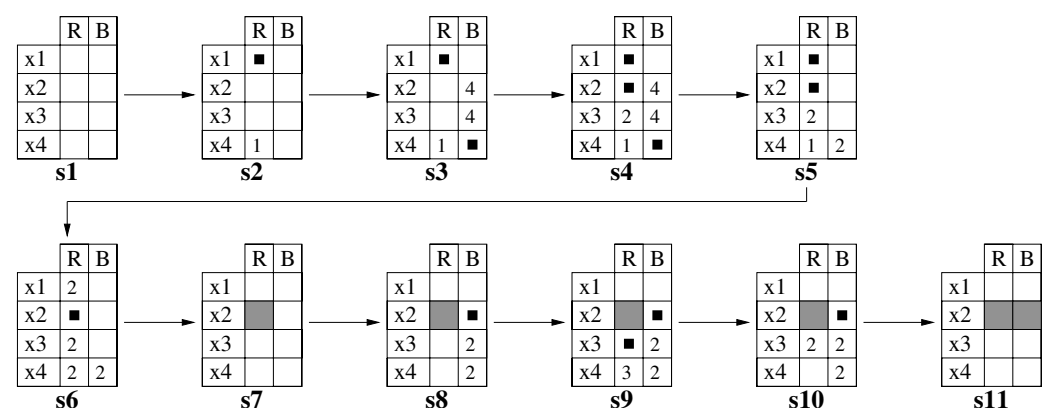

Figure 5. A possible execution of decision repair on $P_{i}$.

It is thus sure that value $B$ for $x_{2}$ does not take part to any solution and can be removed from its domain. Because the domain of $x_{2}$ is now empty, inconsistency of $P_{i}$ is proven.

\section{About the HeURistics For the CHOICE OF THE VARIABLE TO ASSIGN AND THE VALUE TO ASSIGN TO IT}

\subsection{Choice of the VARiable to ASSign}

The heuristics that are used to choose the next variable to assign in case of local consistency of the current partial assignment have two objectives:

- to reduce the width of the tree to explore ${ }^{2}$; this result is achieved by considering first the variables the current domain of which is the smallest; indeed, if we have a set of variables with various domain sizes, the minimal enumeration tree in terms of number of internal nodes is obtained by ordering variables according to an increasing domain size;

- to reduce the depth of the tree to explore; this result is achieved by considering first the variables that are the most constrained, for example that are involved in the greatest number of constraints or in the tightest constraints, in order to prove subproblem inconsistency as soon as possible and thus to cut the enumeration tree as high as possible.

Both objectives can be combined in a heuristic such as dom/deg: to choose a variable the ratio of which between the size of the current domain and the degree in the constraint graph is the smallest [2].

\footnotetext{
2 Speaking of a tree is abusive here, because decision repair does not explore a tree, but a graph nodes of which are partial assignments and edges of which connect two partial assignments when it is possible to go from one to the other via either an assignment, or an unassignment. But the graph of decision repair and the tree of a classical backtrack search have the same set of nodes: the set of possible partial assignments. The only difference is in the set of edges which is much larger with decision repair because of the larger freedom of unassignment: with backtrack search, unassignment must be performed in the inverse order of the asignment order.
} 


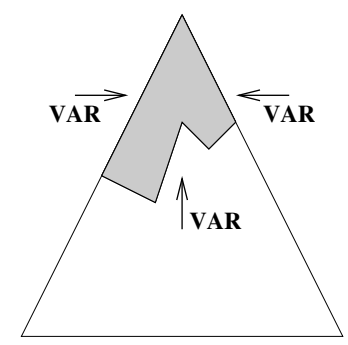

$\overrightarrow{V A R}:$ choice of the next variable to assign

Figure 6. Objectives of the variable heuristics on inconsistent problems.

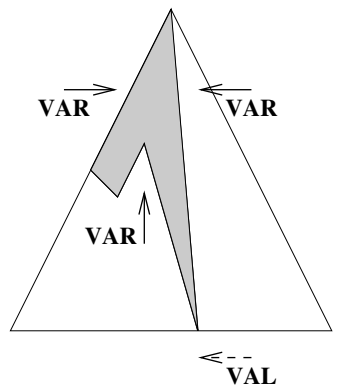

\section{VAL: choice of the value to assign it}

$\overrightarrow{V A R}$ : choice of the next variable to assign

FiguRE 7. Objectives of the variable and value heuristics on consistent problems.

This kind of heuristic is a sensible choice whatever the nature of the problem to solve is: consistent or not. In case of inconsistency, it reduces the size of the tree to explore (see Fig. 6). In case of consistency, it reduces the size of the part of the tree to explore before finding a solution (see Fig. 7).

\subsection{Choice of the value}

If we consider now the heuristics that are used to choose the value to assign to the variable that has been chosen for assignment, they have only one objective:

- to reduce the part of the tree to explore before finding a solution (see Fig. 7); this result is achieved by considering first the values that can the most likely extend the current partial assignment into a solution, in order to prove problem consistency as soon as possible.

In case of inconsistency, such heuristics have no utility, because no solution exists. It results in no gain, but no cost, unless it needs too much computing. In case of consistency, it may result in some gain. Thus, it is a sensible choice whatever the nature of the problem to solve is: consistent or not. 


\section{About the heuristics For the Choice of the VARIABle TO UNASSIGN}

The landscape is different if we consider the heuristics to use to choose the variable to unassign in the current inconsistency explanation: a topic which has not been really explored until now because usual tree search algorithms do not offer such a freedom.

If the problem is consistent, the objective is to build a solution as quickly as possible. In case of inconsistency of the current partial assignment, the objective is thus to identify and to undo bad choices in order to allow the algorithm to make better ones.

Conversely, if the problem is inconsistent, the objective is to build a proof of inconsistency, that is an empty inconsistency explanation, which contains no variable assignment. This result can be achieved if and only if a variable has an empty domain and the removal explanations of all its values are themselves empty (see for an example Fig. 5 in Sect. 2.4). The objective is thus to empty domains with an as small as possible union of the value removal explanations. Note that this result is implicitly achieved in usual depth first tree search when all the values of the first assigned variable have been tried without any success. With decision repair, we want to achieve the same objective using a less ordered search and explicit value removal explanations.

If we try to list the heuristics that could be used to build a solution, we get the following ones:

(1) to choose randomly among the assigned variables, in order to diversify the search, as usually done in local searches [1];

(2) to choose a variable of maximum current domain size, because it offers more space for value choice;

(3) to choose a variable of minimum degree in the constraint graph, for similar reasons;

(4) to choose a variable that is involved in the greatest number of value removal explanations, because its unassignment allows many values to be restored in the domains of the other variables, unless these values must be maintained removed because of other assignments;

(5) more generally, to choose a variable such that the subproblem resulting from its unassignment is the least constrained, using any measure of constrainedness;

(6) to choose the least recently assigned one, because first choices are generally the least informed, and thus the most questionable;

(7) if a note can be associated with any value of any variable, measuring for example its likelihood of taking part in a solution, to choose a variable the assignment of which has the worst note;

(8) in the same conditions, to choose a variable the assignment of which is the most doubtful, for example such that the difference between the note of 
the current assignment and the one of the best assignments in the current domain (alternative choices) is the smallest.

Note that heuristics $2,3,4$, and 5 aim at recovering space for future assignments and that heuristics 6,7 , and 8 aim at undoing bad or doubtful choices.

If we try now to list the heuristics that could be used to build a proof of inconsistency, we get the following ones:

(1) to choose a variable of minimum current domain size, because we are with such a variable closer to an inconsistency, the explanation of which is empty or not;

(2) to choose a variable of maximum degree in the constraint graph, for similar reasons;

(3) to choose a variable such that the subproblem resulting from its unassignment is the most constrained, using any measure of constrainedness;

(4) to choose the most recently assigned one, because last choices remove generally fewer values than first ones do and thus undoing them compels the algorithm to destroy fewer value removal explanations, if we make the assumption of an algorithm which removes irrelevant value removal explanations, that are inconsistent with the current partial assignment, in order to save memory space;

(5) to choose a variable that is involved in the smallest number of value removal explanations, in order to destroy the fewest of them (with the same assumption about the algorithm behaviour), each explanation being eventually weighted by the cost of its production (a production via backtrack is a priori more costly than a direct production via local consistency enforcing) divided by the number of involved variables (a small explanation is more valuable than a large one);

(6) to choose a variable such that, after unassignment, the number of variables involved in the union of its value removal explanations, which can be seen as an inconsistency explanation in progress, eventually divided by the number of removed values, is the smallest, in order to get closer to an empty inconsistency explanation.

Note that heuristics 1, 2, and 3 aim at producing inconsistency as soon as possible, whereas heuristics 4 and 5 aim at maintaining as much as possible existing value removal explanations, and heuristic 6 aims at producing small inconsistency explanations.

Note also that heuristics $1,2,3,4$, and 5 for inconsistent problems are the exact opposites of heuristics $2,3,5,6$, and 4 for consistent problems. If we know nothing about the nature of the problem to solve (consistent or not), this suggests a strategy which would consist to run in parallel two searches with two different goals (to build a solution and to build an inconsistency proof) and thus two different heuristics for the choice of the variable to unassign. But, if we conjecture that the problem is consistent (resp. inconsistent), this suggests another strategy which would consist first to try to build a solution (resp. an inconsistency proof) and 
then to switch the search goal, or to work towards both in parallel, if the previous one has not been achieved by a given deadline.

\section{EXPERIMENTS}

Although we carried out experiments on many problems and instances, with many algorithmic variants, we report in this paper only the ones that have been carried out on randomly generated, but not completely homogeneous, binary $\mathrm{CSPs}^{3}$, with a limited number of algorithmic variants, some of them dedicated to consistent instances and others dedicated to inconsistent ones.

\subsection{Problem instances}

We considered binary CSPs, randomly generated with the usual four parameters: number of variables $n$, domain size $d$ (the same for all the variables), graph connectivity $p_{1}$, and constraint tightness $p_{2}$ (the same for all the constraints), but we broke their homogeneity by partitioning the set of variables into $n c$ clusters of the same size and by introducing a graph connectivity $p_{1}$ inside each cluster (the same for all the clusters) and a lower one $p_{1 c}$ between clusters.

The experimental results that are shown in Figures 8 and 9 have been obtained with $n=50, d=15, n c=5, p_{1}=30, p_{1 c}=20, p_{2}=\frac{100 \cdot n p}{d^{2}}=\frac{100 \cdot n p}{225}$, and $n p$ varying between 81 and 93 by step of 1 around the complexity peak, that is $p_{2}$ varying between 36 and 41.3. 10 instances have been generated for each value of $n p$. For $n p \leq 87\left(p_{2} \leq 38.7\right)$, nearly all the generated instances were consistent and we only considered them (no inconsistent instances). Inversely, for $n p \geq 88$ $\left(p_{2} \geq 39.1\right)$, nearly all the generated instances were inconsistent and we only considered them (no inconsistent instances).

\subsection{Algorithms}

The algorithms we compared are backtrack (BT), conflict directed backjumping (CBJ), dynamic backtracking (DBT), min conflicts (MC), and three variants of decision repair: DR(rand), DR(mostdoubt), and DR(mindestroy).

Except MC, all these algorithms perform forward checking. Except MC and $\mathrm{BT}$, all of them compute and record value removal explanations, and maintain only those that remain relevant. Forward checking is only performed on the unassigned variables and on their current domains. Except MC, all of these algorithms give priority for assignment (resp. unassignment) to the variable that has been

\footnotetext{
3 The choice of focusing experiments on this kind of problems is that classical random CSPS are too homegeneous to exhibit the benefits of methods that turn to advantage the explicit or implicit structure of the instance to solve. For example, all the variables in a classical randomly generated instance have more or less the same degree. Although less systematic, the experiments we carried out on Latin square completion problems, another kind of structured problems [7], produced results that are similar to the ones we show in this section.
} 
unassigned (resp. assigned) just before (see Sect. 2.3). Except for MC, the assignment heuristic consists in choosing an unassigned variable of smallest ratio between its current domain size and its degree in the constraint graph. Except for $\mathrm{MC}$ and the second variant of DR (DR(mostdoubt)), the value heuristic is random. The three variants of DR have in common the choice of the variable to unassign inside the current inconsistency explanation, but differ in the way of making this choice $^{4}$ :

- DR(rand) makes it randomly with a uniform probability distribution over the current inconsistency explanation;

- DR(mostdoubt) uses the results of the pre-computing, for each value of each variable, of the number of values it removes in the domains of the other variables; values are ordered in each domain according to an increasing value of this number; the first value in each current domain is chosen for assignment; the doubtful nature of an assignment is measured by the difference between the number associated with the first value and the one associated with the second one; a variable the assignment of which is the most doubtful (such that the difference is the smallest) in the current inconsistency explanation is chosen for unassignment, with random tiebreaking; such a heuristic clearly aims at producing solutions by removing doubtful assignment choices;

- DR(mindestroy) uses weights that are associated with each assigned variable; when assigned, the weight of a variable $v$ is initialised with the number of values its assignment removes in the domains of the not assigned variables; when unassigned, its weight is reset to 0 and its previous weight is equally distributed among the variables in the current inconsistency explanation that are different from $v$; a variable the weight of which is the smallest in the current inconsistency explanation is selected, with random tie-breaking; this heuristic clearly aims at producing inconsistency proofs by keeping recorded as long as possible value removal explanations which result either from constraint propagation or from backtrack and may have been generated thanks to a lot of computation.

\subsection{EXPERIMENTAL RESUlts}

Because all the considered algorithms involve random choices, each of them has been run 20 times on each instance. Each run has been given a maximum CPUtime of 1200 seconds. A CPU-time of 1200 seconds is associated with any run which did not finish by the deadline. Figure 8 reports the median CPU-time as a function of $p_{2}$, on consistent and then on inconsistent instances. Figure 9 reports the percentage of runs which did not finish by the deadline, among all the runs

\footnotetext{
4 The ways of choosing the variable to unassign inside the current inconsistency explanation used in these three variants are, among all we have experimented, the ones that exhibit a behaviour that is significantly better than the one of classical algorithms at least on a significant constraint thightness interval: before, at, or after the consistency/inconsistency frontier.
} 

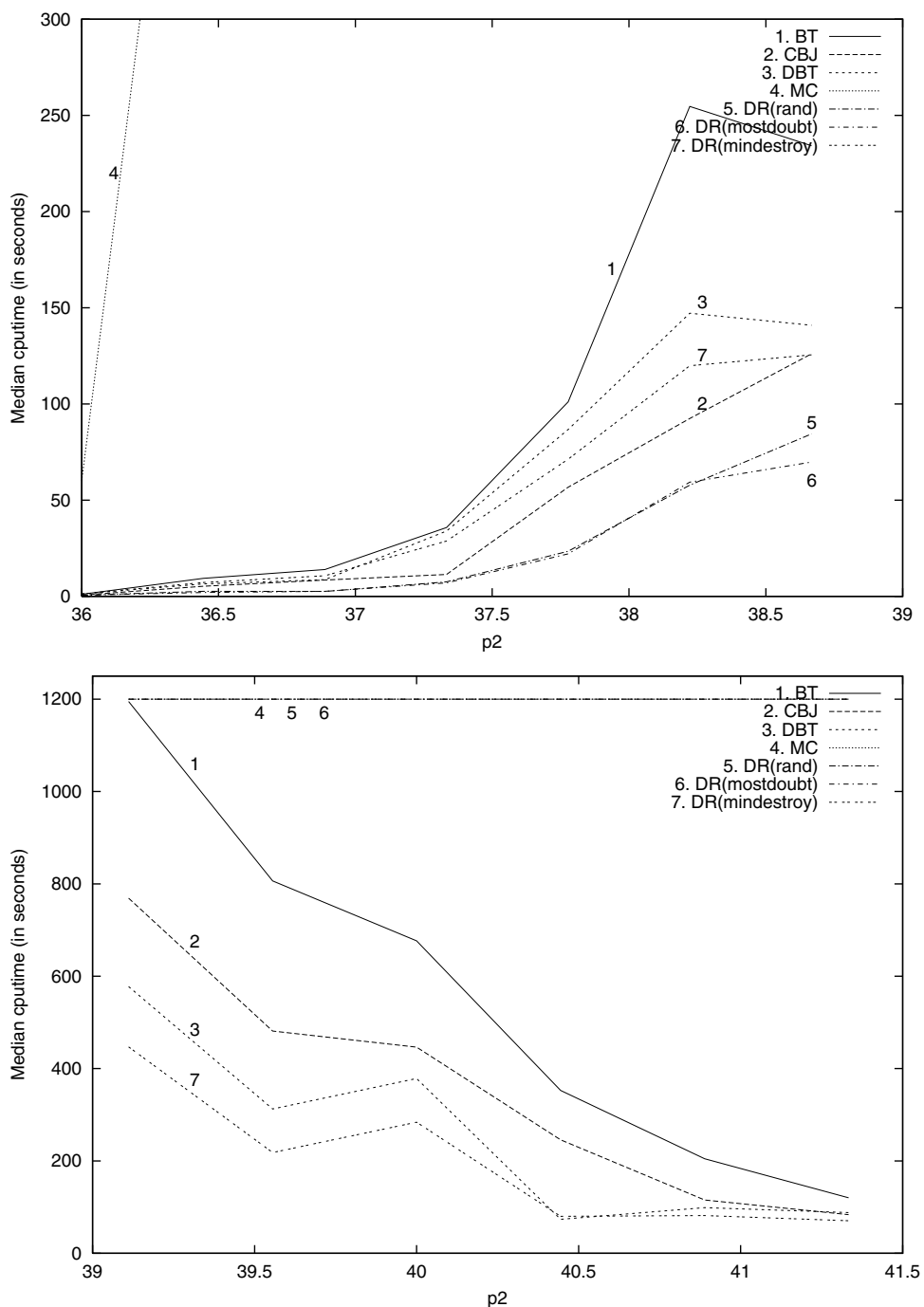

Figure 8. Median CPU-time on randomly generated consistent (top) and inconsistent (bottom) problem instances. Note that scales are different for sake of readability.

on all the instances associated with each value of $p_{2}$, on consistent and then on inconsistent instances. These results allow us to make the following observations.

- If MC may be efficient on consistent instances, it becomes quickly inefficient when approaching the consistency/inconsistency frontier; it is, as other classical local search algorithms, unable to solve inconsistent instances. 

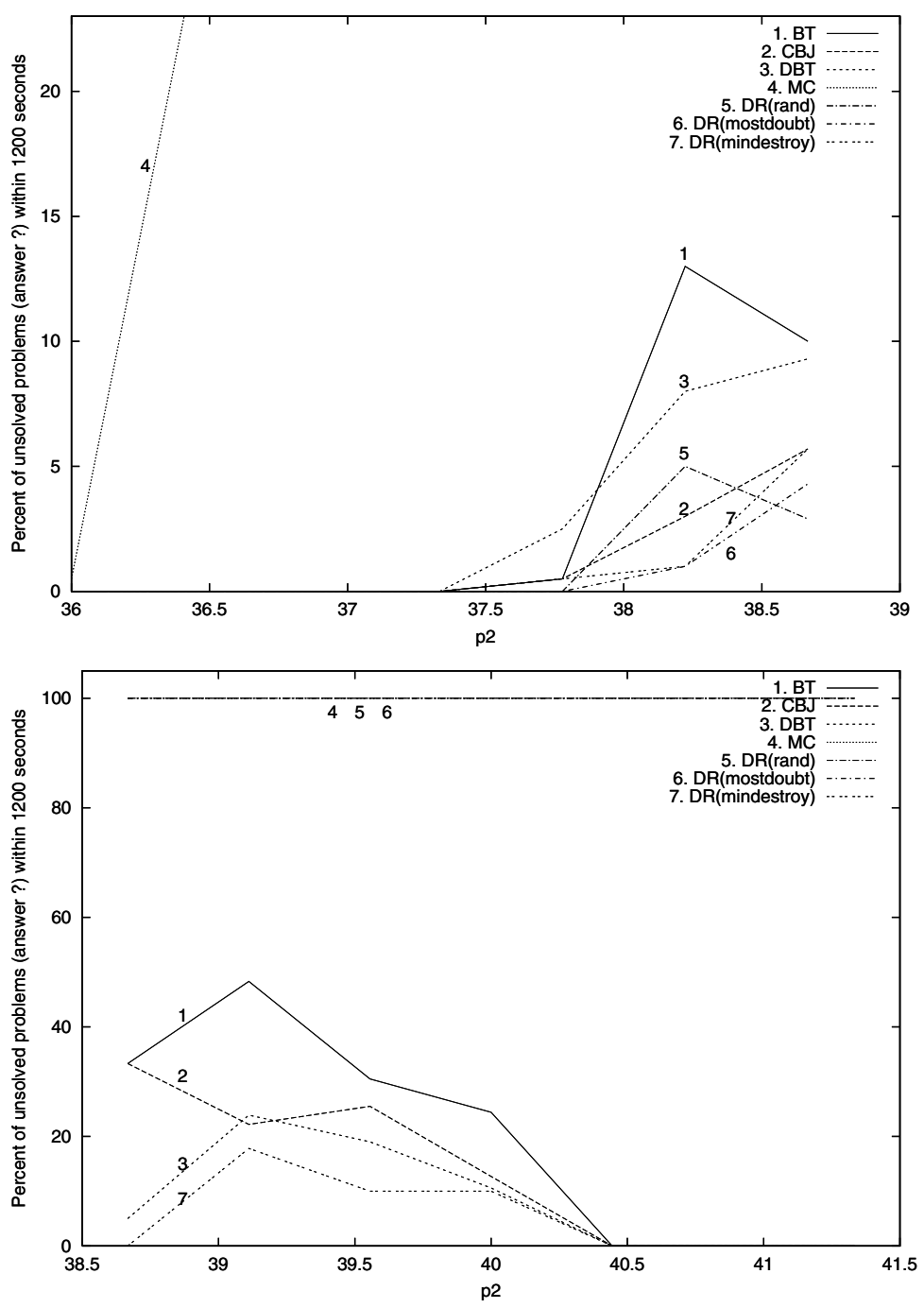

Figure 9. Number of unsolved consistent (top) and inconsistent (bottom) problem instances within 1200 seconds. Note that scales are different for sake of readability.

- BT, CBJ, and DBT present the same usual behaviour with a peak of complexity at the consistency/inconsistency frontier; results of CBJ and DBT are clearly better than those of BT on consistent and inconsistent instances; on consistent instances, CBJ performs better than DBT, but situation is opposite on inconsistent instances. 
- DR(rand) and DR(mostdoubt) produce practically identical results, with only a small advantage to the second one; their global behaviour is similar to that of MC; although they are the most efficient on consistent instances until the consistency/inconsistency frontier, they are, as MC and other classical local search algorithms, unable to solve inconsistent instances.

- Although it is basically a local search algorithm in the space of partial assignments, and thus a priori incomplete, DR(mindestroy) presents the same behaviour as do complete algorithms such as BT, CBJ, and DBT; moreover, it is the most efficient on inconsistent instances until the consistency/inconsistency frontier.

\section{Sufficient CONDitions of COMPLETEness}

These experimental results show that the behaviour of some instances of decision repair, such as DR(mindestroy), is similar to the one of complete algorithms such as backtrack, conflict directed backjumping, and dynamic backtracking. It exhibits the same easy-hard-easy pattern and is clearly different from the one of incomplete algorithms such as min conflicts. Hence, a question arises: are these instances in fact complete?

One can first note that dynamic backtracking, which is an instance of decision repair where the variable to unassign is systematically the most recently assigned one in the current inconsistency explanation, has been proven to be complete [5]. Moreover, other instances of decision repair where only partial order conditions must be met between the variable to unassign and the other variables in the current inconsistency explanation (partial order and general partial order backtracking) have been proven to be complete too $[3,6]$. One the other hand, incomplete dynamic backtracking, which can be seen as a variant of decision repair where the variable to unassign is chosen either randomly or using any heuristic is clearly incomplete [11].

This suggests that some freedom in the choice of the variable to unassign is not incompatible with completeness, but that some restrictions of this freedom are necessary to guarantee it. In this section, we consider two kinds of restrictions that are sufficient to guarantee completeness and generalise the result of completeness of dynamic backtracking. Note that these restrictions are sufficient, but not necessary to guarantee completeness, and that other restrictions could offer the same guarantee.

First, we assume that a weight $w(v, k)$ can be associated with each variable $v$ at each step $k$ of the algorithm. A step is associated with each passing through the main repeat loop of the algorithm with a locally consistent or inconsistent partial assignment (variable bool set to true or false; see Fig. 1). Then, we assume that these weights meet the following initialisation and updating rules:

(1) at $k=0$, all the weights are initialised to 0 ;

(2) if a variable $v$ is assigned at step $k$, its weight at step $k+1$ is set to $\alpha(v, k)$ and the weights of all the other variables are not modified; 
(3) if a variable $v$ is unassigned at step $k$, its weight at step $k+1$ is set to 0 , the weight of each assigned variable $v^{\prime}$, involved or not in the current inconsistency explanation, is updated by adding $\beta\left(v^{\prime}, k\right)$ to it, but the weights of the not assigned variables are not modified.

Note that, at each step $k$, the weights of the not assigned variables are null. The quantity $\alpha(v, k)$ represents the importance or the confidence that can be associated with the assignment of a variable $v$ at step $k$. The quantity $\beta\left(v^{\prime}, k\right)$ represents the change in the importance or the confidence associated with the assignment of a variable $v^{\prime}$, resulting from the unassignment of another variable $v$ at step $k$, in other words the influence of $v$ 's unassignment on $v^{\prime}$ 's weight.

We assume that the decision repair algorithm chooses systematically the variable to unassign among the ones of smallest weight in the current inconsistency explanation, that is among the ones the assignment of which is the least important or reliable. We assume also that, if there are more than one variable of smallest weight in the current inconsistency explanation, the one to unassign is freely chosen, for example randomly with a uniform distribution. In such conditions, Theorems 6.1 and 6.2 can be proven.

Theorem 6.1. If (1) $\forall k, \forall v,[\alpha(v, k) \geq 0] \wedge[\beta(v, k) \geq 0] \wedge[w(v, k) \leq w M a x]$ and (2) $\forall k, \forall v, \forall v^{\prime},\left[v \neq v^{\prime}\right] \wedge\left[v, v^{\prime}\right.$ assigned at step $\left.k\right] \Rightarrow\left[\left|w(v, k)-w\left(v^{\prime}, \bar{k}\right)\right| \geq d M i n>\right.$ $0]$, then decision repair is complete.

In other words, if $\alpha$ and $\beta$ are positive or null, if weights have an upper bound $w M a x$, and if the distance between the weights of two assigned variables has a lower bound $d M i n>0$, then decision repair is complete.

Using a more symbolic approach, Theorem 6.1 could be reformulated as follows: if each variable is valued using a finite and totally ordered valuation set $E$, if the valuation of each not assigned variable is the minimum element of $E$, if the valuation of a variable cannot decrease unless it is unassigned, and if two assigned variables cannot be equally valued, then decision repair is complete.

One can observe that dynamic backtracking meets these conditions by simulating it using the following values for $\alpha$ and $\beta$ : if $v$ is assigned at step $k, \alpha(v, k)$ is equal to the number of not assigned variables at step $k$; if $v$ is unassigned at step $k, \beta(v, k)$ is equal to 1 for all the assigned variables the weight of which is smaller than $v$ 's weight and equal to 0 for all the other assigned variables. It is easy to show that the weight of each assigned variable is an integer between 1 and the number $n$ of variables, that two assigned variables cannot have the same weight, and that the variable to unassign is systematically the most recently assigned in the current inconsistency explanation. But dynamic backtracking can be generalised without forsaking completeness by using any other criterion (different from the number of not assigned variables) to set $\alpha(v, k)$ when assigning $v$ at step $k$.

It also possible to use any criterion as a first criterion and the assignment order as a second criterion by using the following values for $\alpha$ and $\beta$ : if $v$ is assigned at step $k$, if the valuation of $v$ (using the first criterion) is equal to any number $e$ between 1 and $e$ Max, and if $m$ is the number of already assigned variables the valuation of which (using the same first criterion) was equal to $e$, then $\alpha(v, k)$ 
is equal to $e-\frac{m}{n}$; if $v$ is unassigned at step $k, \beta\left(v^{\prime}, k\right)$ is equal to $\frac{1}{n}$ for all the assigned variables $v^{\prime}$ the valuation of which is equal to $v$ 's valuation and the weight of which is smaller than $v$ 's weight, and equal to 0 for all the other assigned variables. Conditions of Theorem 6.1 are still met, with $w$ Max $=e M a x$ and $d$ Min $=\frac{1}{n}$. Completeness is thus guaranteed.

Theorem 6.2. If (1) $\forall k, \forall v,[0 \leq \alpha(v, k) \leq \alpha \operatorname{Max}] \wedge[0 \leq \beta(v, k) \leq f(w(v, k))]$, $f$ being a continuous, positive, and strictly increasing function, and

(2) $\forall k, \forall v,[[v$ unassigned at step $k] \wedge[|I E(k)|>1]] \Rightarrow\left[\beta\left(v^{\prime}, k\right) \geq \beta\right.$ Min $\left.>0\right]$ for at least one variable $v^{\prime}$ of minimum weight in $I E(k)-\{v\}$, then decision repair is complete.

In other words, if $\alpha$ and $\beta$ are positive or null, if $\alpha$ has an upper bound $\alpha M a x$, if $\beta(v, k)$ has an upper bound of the form $f(w(v, k)), f$ being a continuous, positive, and strictly increasing function, and if, when a variable $v$ is unassigned at step $k$ and is not alone in the current inconsistency explanation $I E(k)$, the weight of one of the variables in $I E(k)-\{v\}$ of minimum weight, is increased by at least $\beta M$ in $>0$, then decision repair is complete.

In Theorem 6.2 , the condition of Theorem 6.1 of bounded weights is replaced by conditions of bounded $\alpha$ and $\beta$ : a unique bound for $\alpha$ and a bound which depends on the current weight of the concerned variable for $\beta$. Moreover, the condition of Theorem 6.1 of a minimum distance between the weights of two assigned variables is replaced by the condition of a minimum increase in the weight of at least one variable of minimum weight in $I E(k)-\{v\}$, when $v$ is unassigned at step $k$ and is not alone in $I E(k)$. Note that $v$ has been already chosen to be unassigned among the variables of minimum weight in $I E(k)$. Its weight at step $k+1$ is then set to 0 . Moreover, if $|I E(k)|>1$ ], the weight of at least one variable of minimum weight in $I E(k)-\{v\}$ is increased by at least $\beta$ Min $>0$. Note also that two assigned variables may have now the same weight.

Let us consider for example the following way of setting $\alpha$ and $\beta$ : if $v$ is assigned at step $k, \alpha(v, k)$ is equal to the number of values the removal of which results from $v$ 's assignment; if $v$ is unassigned at step $k$, if $I E(k)$ is the current inconsistency explanation, then $\beta\left(v^{\prime}, k\right)$ is equal to $\frac{w(v, k)}{|I E(k)-1|}$ for all the variables $v^{\prime}$ in $I E(k)$ that are different from $v$, and equal to 0 for all the other assigned variables. In case of assignment, the more removed values, the higher weight. In case of unassignment, the weight of the unassigned variable is equally distributed among the other variables involved in the current inconsistency explanation. It is lost if the unassigned variable is alone in the current inconsistency explanation. Finally, the variable to unassign is roughly speaking chosen among the variables in the current inconsistency explanation that remove either directly (via constraint propagation) or indirectly (via backtrack) the smallest number of values in the domains of other variables. This is the heuristic we use in the algorithm DR(mindestroy) the experimental results of which are shown in Section 5. This name is justified by the fact that this heuristic tries to maintain as much as possible the removals and removal explanations that have been built so far, in order to build a problem inconsistency proof. It is easy to show that conditions of Theorem 6.2 are met by this heuristic, 
with $\alpha$ Max $=(n-1) \cdot d$ (maximum number of values removals resulting from one assignment, if $n$ is the number of variables and $d$ the maximum domain size), $f$ the identity function (because, in the best case, there is only one variable in the current inconsistency explanation other than the unassigned variable $v$ and because $v$ 's weight is the smallest one in the current inconsistency explanation), and $\beta$ Min $=\frac{1}{n-1}$ (because, in the worst case, a weight of 1 is equally distributed among all the other variables). Completeness is thus guaranteed.

To prove both theorems, it must be first noted that there are only two termination cases for the decision repair algorithm: either a complete consistent assignment which is a proof of consistency, or an empty inconsistency explanation which is a proof of inconsistency. So, to prove algorithm completeness, it suffices to prove that it terminates. Termination proofs can be found in a longer version of this paper available at http://www.laas.fr/ cpralet/RAIRO-complete-paper.pdf. Roughly speaking, the proof of Theorem 6.1 uses the fact that weights are bounded. The proof of Theorem 6.2 is more complex because weights are no more assumed to be bounded. The sketch of the proof is the following one: let us assume that the algorithm does not terminate; according to a first lemma (Lem. 1), $\exists k_{0} \geq 0$ such that no definitive value removal occurs after step $k_{0}$; according to a second lemma (Lem. 7), $\forall A, \exists k_{1}>k_{0}$ such that the weight of at least one variable is strictly greater than $A$ at step $k_{1}$; then, according to a third lemma (Lem. 9), we can infer that $\forall i, 1 \leq i, \leq n, \forall A, \exists k_{1}>k_{0}$ such that the weights of at least $i$ variables are strictly greater than $A$ at step $k_{1}$; this applies for $i=n$; thus, $\forall A, \exists k_{1}>k_{0}$ such that the weights of all the variables are strictly greater than $A$ at step $k_{1}$; but, $\alpha M a x$ is an upper bound on the minimum weight over all the variables because, in case of a partial assignment, the weight of at least one variable is null and because, in case of a complete assignment, the weight of the last assigned variable is smaller than or equal to $\alpha M a x$; setting $A>\alpha M a x$ results in a contradiction; as a consequence, the algorithm terminates.

It must be however stressed that, although completeness is guaranteed, the worst-case solving time of decision repair, equipped with such heuristics, is not the same as the one of dynamic backtracking. It is in fact higher. If a search state is characterised by a partial assignment and a set of value removals from the domains of the not assigned variables, because of its termination property, no search state is visited twice by dynamic backtracking. The same property holds with decision repair, when heuristics satisfy conditions of Theorems 6.1 or 6.2 , but with a search state now characterised by a partial assignment, a set of weights associated with the assigned variables, and a set of value removals from the domains of the not assigned variables. This implies that the worst-case solving time is roughly speaking multiplied by the maximum number of weight combinations. This number may be huge and depends on the values of wMax and $d M i n$ in Theorem 6.1, and on the value of $\alpha M a x$ and on the function $f$ in Theorem 6.2.

In fact, we hope that in spite of its higher worst-case solving time, decision repair will be able to exploit its larger freedom in the choice of the variable to 
unassign and to exhibit globally lower mean value and variance of the solving time. Experimental results of Section 5 show that this may be the case.

\section{LOOKING FURTHER}

Beyond these first results, many questions remain unanswered and need further theoretical and experimental studies. Among them:

- Can we define other or weaker sufficient conditions of completeness for decision repair algorithms? Can we better define necessary conditions of completeness?

- What can be the actual benefit of a strategy which would consist to run in parallel two searches: one aiming at building a solution, another one aiming at building an inconsistency proof? Could both searches benefit to each other, for example by exchanging locally consistent partial assignments or value removal explanations?

- What is the influence of the level of local consistency, checked on each partial assignment, on the efficiency of this kind of local search (forward checking, arc consistency...), and, beyond that, the precise influence of all the parameters listed in Section 2.3?

Acknowledgements. This study has been initiated when both authors were working at ONERA, Toulouse, France. Many thanks to Narendra Jussien, from École des Mines de Nantes, France, and Olivier Lhomme, from ILOG, Sophia-Antipolis, France, for the decision repair algorithm, and particularly to Narendra Jussien and Hadrien Cambazard, from École des Mines de Nantes too, for fruitful discussions about this algorithm.

\section{REFERENCES}

[1] E. Aarts and J. Lenstra, Eds. Local Search in Combinatorial Optimization. John Wiley \& Sons (1997).

[2] C. Bessière and J.C. Régin, MAC and Combined Heuristics: Two Reasons to Forsake FC (and CBJ?), in Proc. of the 2nd International Conference on Principles and Practice of Constraint Programming (CP-96)). Cambridge, MA, USA. Lect. Notes Comput. Sci. (1996) 61-75.

[3] C. Bliek, Generalizing Partial Order and Dynamic Backtracking, in Proc. of the 15th National Conference on Artificial Intelligence (AAAI-98). Madison, WI, USA (1998) 319-325.

[4] R. Dechter and R. Mateescu, Mixtures of Deterministic-Probabilistic Networks and their AND/OR Search Space, in Proc. of the 20th International Conference on Uncertainty in Artificial Intelligence (UAI-04). Banff, Canada (2004).

[5] M. Ginsberg, Dynamic Backtracking. J. Artif. Intell. Res. 1 (1993) 25-46.

[6] M. Ginsberg and D. McAllester, GSAT and Dynamic Backtracking, in Proc. of the 4th International Conference on the Principles of Knowledge Representation and Reasoning (KR-94). Bonn, Germany (1994) 226-237.

[7] C. Gomes and B. Selman, Problem Structure in the Presence of Perturbations, in Proc. of the 14th National Conference on Artificial Intelligence (AAAI-97). Providence, RI, USA (1997). 
[8] N. Jussien and O. Lhomme, Local Search with Constraint Propagation and Conflict-based Heuristics. Artif. Intell. 139 (2002) 21-45.

[9] A. Mackworth, Constraint Satisfaction, in Encyclopedia of Artificial Intelligence, S. Shapiro, Ed. John Wiley \& Sons (1992) 285-293.

[10] C. Pralet and G. Verfailllie, Travelling in the World of Local Searches in the Space of Partial Assignments, in Proc. of the International Conference on Integration of Artificial Intelligence and Operations Research Techniques in Constraint Programming for Combinatorial Optimisation Problems (CP-AI-OR-04). Nice, France (2004) 240-255.

[11] S. Prestwich, Combining the Scalability of Local Search with the Pruning Techniques of Systematic Search. Ann. Oper. Res. 115 (2002) 51-72.

[12] P. Prosser, Hybrid Algorithms for the Constraint Satisfaction Problems. Comput. Intell. 9 (1993) 268-299.

To access this journal online: www.edpsciences.org 